\title{
Espagne. Les professeurs se battent-ils contre des moulins à vent?
}

Études de cas

Juan Carlos González Faraco

\section{OpenEdition}

\section{Journals}

Édition électronique

URL : https://journals.openedition.org/ries/1306

DOI : 10.4000/ries. 1306

ISSN : 2261-4265

\section{Éditeur}

France Education international

Édition imprimée

Date de publication : 1 décembre 2005

Pagination : 90-93

ISSN : 1254-4590

Référence électronique

Juan Carlos González Faraco, « Espagne. Les professeurs se battent-ils contre des moulins à vent ? 》, Revue internationale d'éducation de Sèvres [En ligne], 40 | décembre 2005, mis en ligne le 17 novembre 2011, consulté le 08 juillet 2021. URL : http://journals.openedition.org/ries/1306 ; DOI : https://doi.org/ 10.4000/ries. 1306

Ce document a été généré automatiquement le 8 juillet 2021.

(c) Tous droits réservés 


\title{
Espagne. Les professeurs se battent- ils contre des moulins à vent?
}

Études de cas

\author{
Juan Carlos González Faraco
}

1 Les politiques éducatives réformatrices de la seconde moitié du $\mathrm{XX}^{\mathrm{e}}$ siècle en Europe dans l'Espagne des vingt-cinq dernières années - ont plébiscité le rôle prépondérant des professeurs comme collaborateurs nécessaires à tout changement éducatif. Il suffit de consulter la documentation internationale, comme le fameux rapport Delors de 1996 ou celui plus récent d'Eurydice $(2004)^{1}$ sur les enseignants européens. Dans le prologue, V. Reding, commissaire européenne, n'hésite pas à dire que les «professeurs sont des acteurs clés de toute stratégie qui vise à développer la société et l'économie ». D'après A. Nòvoa ${ }^{2}$, ce type de discours politique contraste avec la pauvreté chronique des décisions pratiques et, par conséquent, donne une image fausse de la profession enseignante.

\section{Le malaise des enseignants}

2 La tentation de fabriquer des images autour de cette profession est par ailleurs très courante. Les professeurs, bien évidemment, créent leurs propres images, bien qu'elles soient beaucoup moins favorables que celles des hommes politiques. La majorité d'entre eux ont tendance à se voir comme des Don Quichotte qui luttent, quasiment en pure perte, contre une machine bureaucratique ou académique qui, depuis ses bureaux situés à une distance respectable des établissements, promeut des réformes purement théoriques. Loin de considérer qu'ils en sont les protagonistes ou les participants, ils se voient comme les grands oubliés du système. Beaucoup se sentent abandonnés à leur triste sort dans un univers scolaire raréfié, où ils naviguent à contre courant, avec la nostalgie des temps heureux où la paix régnait dans les salles de classe. Ils ne voient qu'une issue au chaos actuel : le retour à l'ordre et à l'autorité, et le rétablissement de leur fonction traditionnelle de spécialistes respectables d'une discipline. La réforme scolaire la plus récente en Espagne (Loi de qualité de l'éducation, 2002), lancée par le 
gouvernement conservateur du parti populaire, s'est servie du mécontentement du corps enseignant pour justifier certains changements dans le premier cycle du second degré (éducation scolaire obligatoire), celui qui pose le plus de problèmes dans le système éducatif ${ }^{3}$. Les mesures prises favorisaient les parcours différenciés des élèves par la création d'itinéraires et de branches diversifiées avant la fin de l'enseignement obligatoire, renforçaient le pouvoir du chef d'établissement et durcissaient le système d'évaluation.

3 Il faut rappeler que dans l'élaboration de cette loi, très contestée par l'opposition mais en général bien accueillie par les professeurs, les résultats d'une évaluation générale du système ${ }^{4}$, confiée à un groupe d'experts par le ministère de l'Éducation, et ceux de la première enquête PISA de l'OCDE (2000) qui donnait une image médiocre des élèves espagnols, ont joué un rôle important. La publication de la deuxième enquête (2003), dont les résultats sont encore pires, et la suspension par le nouveau gouvernement socialiste de la Loi de qualité ont relancé le débat, abondamment relayé par les médias, sur le faible rendement et le manque de motivation des élèves, sur les conflits dans les classes et le rôle ingrat des enseignants.

4 La nouvelle équipe ministérielle de gauche, avant de se lancer dans sa propre réforme, a diffusé sur Internet un document pour nourrir le débat, intitulé « Une éducation de qualité pour tous et partagée par tous $»^{5}$, qui se résumait à un inventaire de bonnes intentions. Naturellement, le document insistait sur le "rôle indispensable » des professeurs dans le processus réformateur imminent dont les objectifs sont semblables à ceux que l'on retrouve invariablement dans tous les forums internationaux: améliorer la formation initiale, créer un statut professionnel, faciliter la mobilité professionnelle, améliorer la reconnaissance sociale, etc. Comme on pouvait le prévoir, le nouveau projet de Loi organique de l'éducation (LOE, 2005), promu par les socialistes, consacre un long chapitre aux professeurs et à une meilleure reconnaissance sociale de la fonction enseignante. C'est avec une froideur et une circonspection manifestes que les enseignants accueillent ces «nouveaux» discours, d'autant que les réformateurs actuels ont repris, en partie du moins, le modèle pédagogique promu par les précédents gouvernements socialistes dans les années quatre-vingts et quatre-vingt-dix ${ }^{6}$. Les critiques émises à l'époque par de nombreux professeurs, surtout dans le secondaire, contre ce modèle global et constructiviste, sont toujours d'actualité même si les enseignants se montrent sans doute aujourd'hui moins agressifs. Pour beaucoup, cet apaisement n'a d'autre explication que l'acceptation résignée d'une situation professionnelle qui demeure pénible. C'est le syndrome du «burn out » du professeur.

5 Les récits que les enseignants font de leur profession au quotidien dépeignent un paysage désolé dans lequel se joue un drame avec des « victimes » - eux-mêmes - et des «bourreaux » : des « objecteurs scolaires » qui ne veulent pas apprendre et créent des problèmes de cohabitation; des parents qui se désintéressent de l'éducation de leurs enfants ou qui envahissent leur espace professionnel; des experts qui les utilisent sans ménagement pour leurs expériences pédagogiques ${ }^{7}$; des hommes politiques et une administration qui ne respectent pas leur autonomie professionnelle. Cette vision alimente ce que l'on appelle le "malaise enseignant», un concept assez vague né d'études sur la personnalité et la santé des professeurs. À ces perceptions plus ou moins subjectives viennent s'ajouter de vieux problèmes et des revendications qui n'ont jamais abouti ainsi que quelques indicateurs sociologiques préoccupants ${ }^{8}$ : vieillissement $\mathrm{du}$ corps professoral, future pénurie de professeurs, féminisation 
croissante, prolétarisation ${ }^{9}$ ou professionnalisation à moitié réussie seulement, perte de prestige social, bas salaires, formation inadaptée ${ }^{10}$, surtout dans le secondaire ${ }^{11}$, etc. Les avis divergent quand il s'agit d'en déterminer les causes et les responsabilités, bien qu'on ait tendance le plus souvent à désigner comme principaux responsables divers agents parmi lesquels ne figurent généralement pas les enseignants.

Cependant, des analystes qui adoptent une position tout à fait différente voient dans le «malaise » des professeurs un de ces discours dont ils se servent pour défendre leurs privilèges professionnels. Le sociologue espagnol M. Fernández Enguita (1996), dans un $\operatorname{article}^{12}$ qui fit grand bruit lors de sa parution, mentionne la résistance opiniâtre des enseignants aux tentatives d'innovations de l'administration. Il en conclut que les progrès nécessaires du service public d'éducation se heurtent de front au pouvoir, aux intérêts et aux habitudes du corps enseignant ${ }^{13}$. Le recours à la "victimisation " ou à d'autres tactiques défensives ne serait que l'expression d'un corporatisme masqué. D'après lui, il existe trois causes à cette tentative de "privatisation » corporative d'un espace public tel que l'école : la féminisation de la profession, le manque de vocation des enseignants et l'irresponsabilité accommodante des syndicats.

\section{Une redéfinition professionnelle nécessaire}

7 Pourtant, que l'on considère les enseignants comme des Don Quichotte ou des Sancho Pança, il est intéressant de relever qu'une bonne partie des analyses actuelles tournent autour de la nécessité de redéfinir leur identité. Les arguments utilisés peuvent parfois paraître presque métaphysiques voire anachroniques. Ainsi, dans un bref et récent article intitulé «Être un maitre $~^{14}$, une écrivaine célèbre affirme que l'enseignement n'est pas une profession mais un métier, car enseigner ne revient pas à « exercer » une profession mais bien à vivre une "passion ». Dans ce contexte, on ne s'étonnera pas non plus (même si cela ne laisse pas d'inquiéter) que le psychiatre espagnol C. Castilla del Pino ait utilisé l'expression toute faite "pathologie de l'identité » pour décrire les problèmes de santé les plus fréquents de ses nombreux patients enseignants. D'une façon ou d'une autre, la nécessité de redéfinir la profession enseignante est devenu l'objet prioritaire d'un débat éducatif non dénué de rhétorique et parcouru à l'heure actuelle par des chemins à première vue discordants.

Certains mettent leur espoir dans les nouvelles technologies comme voie de pénétration des innovations didactiques et comme outil de revitalisation de l'école et de ses acteurs. De fait, les nouvelles technologies de l'information et de la communication sont le secteur le plus demandé par les professeurs en formation continue et le plus développé dans les programmes de l'administration destinés à améliorer le système éducatif. Pour beaucoup, le jeu technologique serait le répulsif dont l'École et la profession enseignante auraient besoin après une étape agitée de réformes hautement idéologiques. D'autres, en revanche, parient sur la revitalisation morale et professionnelle du professeur avec l'adoption de mesures améliorant ses conditions de travail et son prestige social, mais aussi grâce à un appel, presque poétique, à l'optimisme ${ }^{15}$ et à l'engagement éthique. Le succès d'un film français comme Être et avoir met en évidence le désir, partagé par un grand nombre, d'affronter le problème de l'école actuelle en prenant la défense de ces valeurs considérées comme désuètes par d'aucuns, plutôt qu'en ayant recours à des innovations techniques ou pédagogiques. 
9 C'est dans ce labyrinthe de discours et d'images superposés qu'évolue aujourd'hui la fonction enseignante. De réforme en réforme, a peu à peu été défini le type de professeur dont on ne veut pas, même si c'est avec quelques désaccords. Il est urgent de nous mettre d'accord maintenant, sur le type de professeur dont nous avons besoin, mais surtout sur les décisions, probablement radicales, qu'il faudra prendre pour le «fabriquer ». Ces décisions seront-elles effectivement prises un jour?

\section{NOTES}

1. EURYDICE (2004). Key Topics in Education in Europe. vol. 3. The teaching profession in Europe: Profile, trends and concern (http://www. eurydice.org).

2. Nòvoa, A. (1999). «La nueva cuestión central de los profesores. Exceso de discursos, pobreza de prácticas ». Cuadernos de Pedagogía, n² 286, pp. 102-108.

3. Vicente Algueró, F. de (2002). «El profesorado en la Ley de Calidad». Revista de Educación, $\mathrm{n}^{\circ} 329$, pp. 67-76.

4. Ministerio de Educación, Instituto Nacional de Calidad y Evaluación (1998). Diagnóstico del Sistema Educativo. La escuela secundaria obligatoria. La profesión docente (vol. 5).

5. http://www.mec.es

6. Ley de Ordenación General del Sistema Educativo (LOGSE, 1990).

7. Ruiz Paz, M. (2003). La secta pedagógica. Madrid, Grupo Unisón de Ediciones.

8. Pérez Gómez A. et al. (2003). «La situación profesional de los docentes. Luces y sombras ». Cuadernos de Pedagogía, n 326, pp. 34-40.

9. Arenas Vázquez C. (2001). «El profesorado de secundaria frente a las reforma ». Cuadernos de Pedagogía, $\mathrm{n}^{\circ}$ 303, pp. 84-86.

10. Ph. Perrenoud argumente en faveur d'un modèle de professionnalisme ouvert pour la fonction enseignante, et par voie de conséquence pour la formation, par opposition au modèle traditionnel de compétence minimum qu'adoptent, dans leur grande majorité, les enseignants du secondaire comme modèle professionnel de référence. Voir «La profesión docente, entre la proletarización y la profesionalización ». Perspectivas, vol. 26, n³ 3, pp. 549-570, 1996.

11. Par exemple, en ce qui concerne la France, A. Van Zanten souligne le manque de cohérence de la formation continue des professeurs du secondaire. Voir «La profession enseignante en France, quelles évolutions? ", Revue internationale d'Éducation Sèvres, n 30, p. 90.

12. Fernández Enguita, M. «Es pública la escuela pública ». Cuadernos de Pedagogía, n²84, pp. 76-81. Voir aussi : «Las profesiones como poder social : el caso del profesorado ». Revista de Educación, n³ 324, pp. 143-154, 2001.

13. Le fait que les enseignants espagnols soient majoritairement des fonctionnaires (74 \%) est essentiel pour comprendre les attitudes défensives des professeurs face à d'autres acteurs du système.

14. Aldecoa J. (2003). «Ser maestro », Cuadernos de Pedagogía, nº 326, p. 41.

15. Santos Guerra Miguel A. (2004). «Invitación al optimismo ». Cuadernos de Pedagogía, n 334, pp. 86-90. 


\section{AUTEUR}

\section{JUAN CARLOS GONZÁLEZ FARACO}

Professeur, Université de Huelva. 